TecnoLógicas

ISSN-p 0123-7799

ISSN-e 2256-5337

Vol. 22, No. 44

Enero-abril de 2019

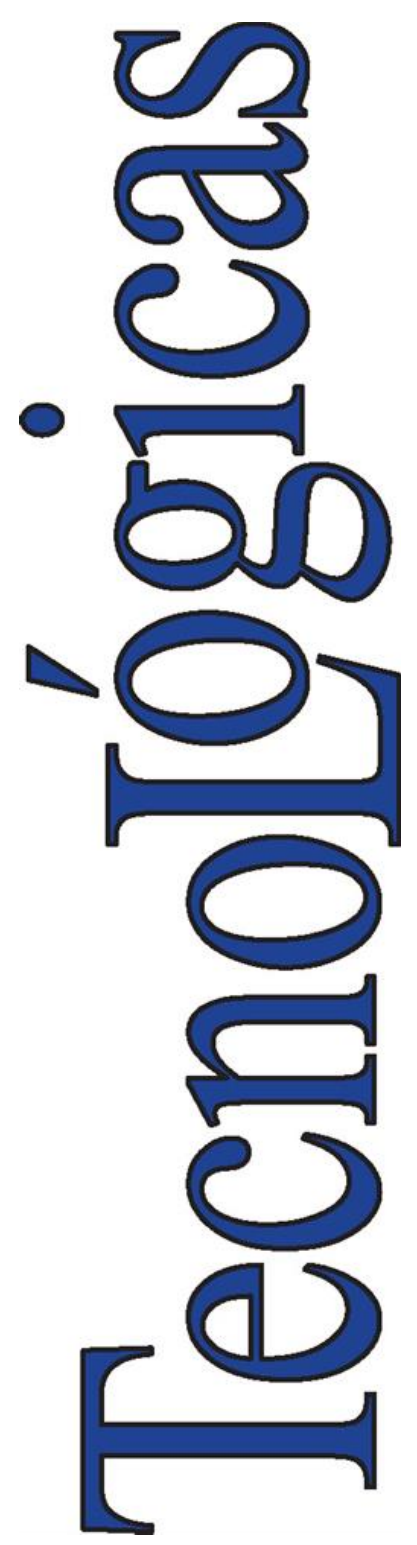

(C) Instituto Tecnológico Metropolitano Este trabajo está licenciado bajo una Licencia Internacional Creative Commons Atribución (CC BY-NC-SA)

\section{Prostasomas: búsqueda de biomarcadores para la detección temprana del cáncer prostático}

\section{Prostasomas: search of biomarkers for the early detection of prostate cancer}

\author{
Manuela Antonia García ${ }^{1}$, Julieth Rueda ${ }^{2}$, y \\ Johanna Pedroza-Díaz ${ }^{3}$
}

Recibido: 02 de junio de 2018

Aceptado: 22 de octubre de 2018

Cómo citar / How to cite

M. A. García, J. Rueda, y J. Pedroza-Díaz, Prostasomas: búsqueda de biomarcadores para la detección temprana del cáncer prostático. TecnoLógicas, vol. 22, no. 44, pp. 131-148, 2019.

https://doi.org/10.22430/22565337.1181

1 Estudiante de ingeniería biomédica, Facultad de Ciencias Exactas y Aplicadas, Instituto Tecnológico Metropolitano, Medellín-Colombia, manuelagarcia189035@correo.itm.edu.co

2 Estudiante de ingeniería biomédica, Facultad de Ciencias Exactas y Aplicadas, Instituto Tecnológico Metropolitano, Medellín-Colombia, juliethrueda188597@correo.itm.edu.co

3 MSc en Ciencias Básicas Biomédicas, Facultad de Ciencias Exactas y Aplicadas, Grupo de Investigación e Innovación Biomédica GI2B, Línea de Ciencias Biomédicas. Microbióloga, Instituto Tecnológico Metropolitano, Medellín-Colombia, ninipedroza@itm.edu.co 


\section{Resumen}

El cáncer de próstata es la segunda enfermedad más diagnosticada en hombres a nivel mundial, con una tasa de mortalidad creciente en los últimos años. Actualmente, se cuenta con dos pruebas de detección temprana: la medición de los niveles en sangre del antígeno prostático específico y el tacto rectal de la próstata. Sin embargo, estas pruebas no presentan óptima especificidad y sensibilidad para su detección. Aunque diferentes estudios han buscado nuevos biomarcadores mediante la implementación de tecnologías, como secuenciación de nueva generación, espectrometría de masas, entre otras, aún persisten las mismas desventajas, por lo que no les ha permitido a estos su uso en la práctica clínica; razón por la cual, el descubrimiento de nuevos biomarcadores para el diagnóstico de cáncer de próstata, constituye un desafío para la comunidad científica.

Los prostasomas corresponden a vesículas extracelulares secretadas por el tejido prostático normal o tumoral que pueden ser detectadas en diferentes fluidos. Estructuralmente, los prostasomas difieren de otros exosomas, por su tamaño, composición de membrana y contenido específico de proteínas, lo que los convierten en una fuente potencial y novedosa de biomarcadores clínicos.

En este contexto, esta revisión presenta un panorama general de los biomarcadores proteicos, aislados desde prostasomas presentes en diferentes fluidos biológicos, para el posible diagnóstico de cáncer de próstata. Para ello se realizó una búsqueda sistemática en PubMed para estudios en proteómica para cáncer de próstata, con criterios como: vesículas extracelulares, exosomas y prostasomas, asimismo, sangre, orina, líquido seminal, entre otras muestras biológicas.

\section{Palabras clave}

Biomarcadores; cáncer de próstata; vesículas extracelulares; prostasomas; proteínas.

\section{Abstract}

Prostate cancer is the second most diagnosed disease among men worldwide, with a growing mortality rate in recent years. Currently, two methods can be used for early detection: prostate-specific antigen blood tests and digital rectal exams. However, their specificity and detection sensitivity are not optimal. Although several studies have searched for new biomarkers by implementing technologies such as next-generation sequencing and mass spectrometry, the same disadvantages persist. For that reason, biomarkers are not allowed in clinical practice and, therefore, discovering new ones to diagnose prostate cancer is a challenge for the scientific community.

Prostasomes are extracellular vesicles secreted by healthy or tumoral prostatic tissue that can be detected in different fluids. Prostasomes are different from other exosomes in size, membrane composition, and specific protein content, which makes them a potential and novel source of clinical biomarkers.

In such context, this review article presents a general overview of protein biomarkers isolated from prostasomes present in different biological fluids for the possible diagnosis of prostate cancer. For that purpose, a systematic search was conducted in PubMed to find proteomic studies regarding prostate cancer using terms such as extracellular vesicles, exosomes, prostasomes, blood, urine, and seminal fluid, among other biological samples.

\section{Keywords}

Biomarkers, Prostate Cancer, Extracellular Vesicles, Prostasomes, Proteins. 


\section{INTRODUCCIÓN}

El cáncer de próstata $(\mathrm{CaP})$ es la segunda neoplasia más común en hombres [1]. Un estimado de 1,1 millones de varones en todo el mundo fueron diagnosticados con CaP en 2012, así mismo, es la quinta causa de muerte asociada a cáncer en los hombres [2]. En nuestro país se diagnostican más de 6.500 casos nuevos y mueren más de 2.400 hombres anualmente [3].

El diagnóstico de $\mathrm{CaP}$ cuenta con dos pruebas de detección temprana, la medición del Antígeno Prostático Específico (PSA) y el tacto rectal de la próstata [4]. El PSA es una proteína producida por la próstata cuya expresión aumentada puede obedecer a distintas causas, entre ellas Hiperplasia Prostática Benigna (HPB), prostatitis [5], edad avanzada [6] y el CaP. A pesar de ser una prueba poco invasiva y sensible [7], presenta baja especificidad, lo cual conduce a falsos positivos para el diagnóstico, de hecho, la probabilidad de diagnosticar $\mathrm{CaP}$ en un paciente con PSA superior a $4 \mathrm{ng} / \mathrm{mL}$ es del $21 \%$, lo que representa una "sobre valoración» del $75 \%$, y un "sobre diagnóstico» que oscila entre 30$50 \%$ en una enfermedad tumoral que podría ser indolente [8]. Incluso algunos pacientes con $\mathrm{CaP}$ se caracterizan por tener bajos niveles de PSA. Con este panorama, en los últimos años se han reportado formas alternativas de la prueba de PSA que tienen un mejor rendimiento, el Índice de Salud Prostática (PHI) es una nueva fórmula que combina las tres formas de PSA (PSA total, PSA libre y proPSA) en una única puntuación que puede ser utilizada para el diagnóstico y manejo de $\mathrm{CaP}$ [9]. Un estudio reciente [10] demostró que los niveles de proPSA y el PHI pueden discriminar entre los pacientes con cáncer de próstata y los que tienen PHB o una inflamación crónica prostática, lo cual reduce la necesidad de biopsias innecesarias en un $27 \%$ de los casos. Sin embargo, el PHI no es eficaz para la estratificación de los pa- cientes, y por lo tanto se ha continuado la búsqueda de mejores biomarcadores que puedan ser usados en el diagnóstico, pronóstico y seguimiento del $\mathrm{CaP}$ [10].

En cuanto al tacto rectal, se tiene que esta prueba genera incomodidad, ya que implica la exploración digital de la glándula a través del recto, además, solo permite palpar la cara anterior de la próstata, por lo que resulta bastante ineficiente, se estima que del 23 al $45 \%$ de los $\mathrm{CaP}$ se pasan por alto y aproximadamente el $50 \%$ se diagnostican en una etapa avanzada [7]. Es por ello que el diagnóstico temprano para CaP resulta fallido en muchos casos. Por otro lado, otros marcadores como el codificado por el gen PCA3 (gen del antígeno tres en cáncer de próstata) que traduce para una proteína exclusiva del tumor prostático, la cual es detectada tanto en la orina como en el fluido prostático de los pacientes han sido propuestos, aproximadamente el $95 \%$ de los pacientes con $\mathrm{CaP}$ exhiben niveles de PCA3 en orina superiores al grupo control saludable. En un par de estudios publicados analizaron la efectividad de este biomarcador, en la detección del $\mathrm{CaP}$ en muestras de orina posterior a un masaje prostático, reportando una sensibilidad del $66 \%$ y una especificidad del $89 \%$ en el primer estudio [11] y en el segundo un $74 \%$ y $91 \%$ de sensibilidad y especificidad respectivamente [12]. Estos resultados obtenidos, suscitaron interés en el desarrollo de una prueba en orina que pudiera estar disponible comercialmente.

El test PROGENSA-PCA3 se basa en la amplificación del ARNm de PCA3 en la orina medida por transcripción. El resultado se traduce en un puntaje que es valorado en el contexto clínico de cada paciente. Esta prueba urinaria de PCA3 usada en conjunto con la prueba sérica de PSA incrementa el umbral de detección del $\mathrm{CaP}$ [13]. Hasta la fecha este es el único biomarcador de orina comercialmente disponible, con ventajas claras como que el volumen de la próstata no afecta el resultado (como en el caso de la prueba sérica de 
PSA), e identifica aquellos pacientes con alto riesgo de desarrollar $\mathrm{CaP}$ y por ende mejora el diagnóstico reduciendo la necesidad de practicar biopsias innecesarias.

Los pacientes sospechosos para $\mathrm{CaP}$ por lo general deben someterse a biopsias de próstata en serie para la vigilancia activa. Sin embargo, el análisis histopatológico puede subestimar el grado o extensión de la enfermedad o incluso no permitir la observación del tejido tumoral, esto debido a la naturaleza multifocal y heterogeneidad de los tumores prostáticos [14]. Por lo tanto, se hace imperativa la búsqueda e implementación de marcadores transportados por sangre, orina $\mathrm{u}$ otros líquidos corporales de fácil obtención, que permitan la detección temprana de la enfermedad con el fin de hacer la vigilancia activa del $\mathrm{CaP}$, de manera menos invasiva, además de reducir costos en el sistema de salud y evitar complicaciones potenciales por el muestreo de tejido prostático a través de la biopsia transrectal.

Mediante estudios In vitro se demostró que todos los tipos de células humanas liberan vesículas extracelulares (VEs) y que por lo tanto es común observarlas en los diferentes fluidos corporales [15]. Diversas funciones han sido atribuidas a dichas VEs liberadas desde células normales, apoptóticas y necróticas, entre ellas, su participación en la comunicación intercelular, particularmente, en procesos de respuesta inmune adaptativa [16]. Adicionalmente, en la última década se ha descrito de manera detallada los mecanismos moleculares involucrados en la formación de las VEs y su composición química.

Los fluidos prostáticos contienen VEs de dos tipos, prostasomas y exosomas: los prostasomas (150-500 $\mathrm{nm})$, son producidos por células epiteliales ductales prostáticas que son un componente normal del líquido seminal y juegan un papel en la fertilidad masculina; y exosomas, que son nanovesículas especializadas (30-100 nm) con una morfología en forma de copa, secretada activamente por una variedad de células normales y tumorales [16]. Se ha encontrado una gran cantidad de exosomas en suero, orina y efusiones tumorales de pacientes con cáncer [16].

Partiendo de que las VEs de las células cancerosas tienen contenidos únicos $\mathrm{y} \mathrm{es}^{-}$ pecíficos del cáncer, junto con la observación de que los prostasomas están presentes tanto en la sangre como en la orina de los pacientes con $\mathrm{CaP}$, surgió la hipótesis de que las VEs pueden proporcionar marcadores útiles para el CaP. Dicha idea fue respaldada por los primeros estudios en los que se compararon los perfiles de proteoma de VEs aisladas de cultivos de líneas celulares tumorales y no tumorales de próstata, en los cuales se identificaron múltiples proteínas que podrían constituir posibles biomarcadores proteicos candidatos para CaP [17]. En este contexto, el objetivo de esta revisión es presentar un estado del arte de los biomarcadores proteicos para diagnóstico/pronóstico de $\mathrm{CaP}$, aislados desde prostasomas y otras VEs. Los biomarcadores revisados son presentados en la Tabla 1, discriminando las ventajas y desventajas de los estudios en los que se reportan y la posible utilidad (ver Tabla 1).

\section{MEtodologíA}

Se realizó una búsqueda sistemática en PubMed para estudios en proteómica para cáncer de próstata, con criterios como: VEs, exosomas y prostasomas, asimismo, sangre, orina, líquido seminal, entre otras muestras biológicas. Después de una revisión manual 39 artículos originales fueron incluidos así, 16 para fluido sanguíneo, 7 para orina, 9 para líquido seminal y 7 para otras fuentes biológicas. la bibliografía fue recopilada entre marzo y julio de 2018 . 
Prostasomas: búsqueda de biomarcadores para la detección temprana del cáncer prostático

Tabla 1. Proteínas asociadas a prostasomas propuestas como posibles marcadores para cáncer de próstata. Fuente: autores.

\begin{tabular}{|c|c|c|c|c|c|}
\hline $\begin{array}{l}\text { Fluido } \\
\text { biológico }\end{array}$ & PROTEÍNA & VENTAJA & DESVENTAJA & UTILIDAD & REF \\
\hline \multirow[t]{3}{*}{ Sangre } & Survivin & $\begin{array}{l}\text { Asociada con la severi- } \\
\text { dad tumoral y la morta- } \\
\text { lidad }\end{array}$ & $\begin{array}{l}\text { Tamaño de mues- } \\
\text { tra pequeño }\end{array}$ & $\begin{array}{l}\text { Diagnóstico/ respuesta al } \\
\text { tratamiento }\end{array}$ & $\begin{array}{l}{[23],} \\
{[28],} \\
{[29]}\end{array}$ \\
\hline & PTEN & $\begin{array}{l}\text { Expresión aumentada } \\
\text { solo en células tumora- } \\
\text { les }\end{array}$ & $\begin{array}{l}\text { Se requieren } \\
\text { mayores estudios }\end{array}$ & $\begin{array}{l}\text { Ayuda en el control del } \\
\text { crecimiento celular }\end{array}$ & [31] \\
\hline & NKG2D & $\begin{array}{l}\text { Asociada con la respues- } \\
\text { ta inmune y la progre- } \\
\text { sión del cáncer }\end{array}$ & $\begin{array}{l}\text { Se requieren } \\
\text { mayores estudios }\end{array}$ & $\begin{array}{l}\text { Tratamientos de inmu- } \\
\text { noterapia }\end{array}$ & [35] \\
\hline \multirow[t]{6}{*}{ Orina } & PSMA & $\begin{array}{l}\text { Presente en las fraccio- } \\
\text { nes de VEs aisladas de } \\
\text { la orina de pacientes con } \\
\text { CaP. }\end{array}$ & $\begin{array}{l}\text { Se requieren } \\
\text { mayores estudios } \\
\text { para aprobar los } \\
\text { hallazgos. }\end{array}$ & $\begin{array}{l}\text { Alto potencial como } \\
\text { biomarcador de } \mathrm{CaP} .\end{array}$ & {$[17]$} \\
\hline & LAMTOR & $\begin{array}{l}\text { Aumento significativo } \\
\text { en secreciones del } \mathrm{CaP}\end{array}$ & $\begin{array}{l}\text { Se requieren } \\
\text { mayores estudios }\end{array}$ & $\begin{array}{l}\text { Alto potencial como } \\
\text { biomarcador de } \mathrm{CaP} \text {. }\end{array}$ & [39] \\
\hline & $\begin{array}{l}\text { TMPRSS2: } \\
\text { ERG }\end{array}$ & $\begin{array}{l}\text { Aumento en CaP. Re- } \\
\text { quiere poca cantidad de } \\
\text { muestra }\end{array}$ & $\begin{array}{l}\text { Se requieren } \\
\text { estudios en gru- } \\
\text { pos poblacionales } \\
\text { de mayor cantidad }\end{array}$ & $\begin{array}{l}\text { Diagnóstico/ vigilancia } \\
\text { tumoral del CaP }\end{array}$ & {$[16]$} \\
\hline & PCA-3 & $\begin{array}{l}\text { Clasificación de la } \\
\text { gravedad del fenotipo } \\
\text { tumoral }\end{array}$ & $\begin{array}{l}\text { Se requieren } \\
\text { estudios en gru- } \\
\text { pos poblacionales } \\
\text { de mayor cantidad }\end{array}$ & $\begin{array}{l}\text { Diagnóstico/ vigilancia } \\
\text { tumoral del CaP/ clasifi- } \\
\text { cación de la enfermedad }\end{array}$ & [16] \\
\hline & Anexina A3 & $\begin{array}{l}\text { Alta especificidad como } \\
\text { biomarcador para } \mathrm{CaP}\end{array}$ & $\begin{array}{l}\text { Complementaria } \\
\text { al PSA }\end{array}$ & Vigilancia epidemiológica & {$[16]$} \\
\hline & TM256 & $\begin{array}{l}\text { Posee una alta sensibi- } \\
\text { lidad para diferenciar el } \\
\mathrm{CaP}\end{array}$ & $\begin{array}{l}\text { Se requieren } \\
\text { estudios en gru- } \\
\text { pos poblacionales } \\
\text { de mayor cantidad }\end{array}$ & $\begin{array}{l}\text { Alto potencial como } \\
\text { biomarcador de } \mathrm{CaP} .\end{array}$ & [39] \\
\hline \multirow[t]{4}{*}{$\begin{array}{l}\text { Liquido } \\
\text { seminal }\end{array}$} & $\begin{array}{l}\text { CD13 y } \\
\text { DPPIV }\end{array}$ & $\begin{array}{l}\text { Abundante concentra- } \\
\text { ción en LS de pacientes } \\
\text { con CaP }\end{array}$ & $\begin{array}{l}\text { Se requieren } \\
\text { mayores estudios }\end{array}$ & $\begin{array}{l}\text { Investigaciones en pros- } \\
\text { tasomas }\end{array}$ & [43] \\
\hline & Galectina-3 & $\begin{array}{l}\text { Asociada a funciones en } \\
\text { la regulación de los } \\
\text { espermatozoides, la } \\
\text { inmunosupresión y la } \\
\text { progresión del CaP. }\end{array}$ & $\begin{array}{l}\text { Se requieren } \\
\text { mayores estudios }\end{array}$ & Progresión del CaP & [45] \\
\hline & $\begin{array}{l}\text { Cromogra- } \\
\text { nina A }\end{array}$ & $\begin{array}{l}\text { Expresada significati- } \\
\text { vamente en prostaso- } \\
\text { mas y VEs de células } \\
\text { metastásicas }\end{array}$ & $\begin{array}{l}\text { Se requieren } \\
\text { mayores ensayos }\end{array}$ & $\begin{array}{l}\text { Grado de malignidad del } \\
\text { cáncer }\end{array}$ & {$[47]$} \\
\hline & $\begin{array}{l}\text { Quinasas y } \\
\text { ATPasas }\end{array}$ & $\begin{array}{l}\text { Actividad aumentada } \\
\text { significativamente: } \\
\text { fosforilación }\end{array}$ & $\begin{array}{l}\text { Se requieren } \\
\text { mayores ensayos }\end{array}$ & $\begin{array}{l}\text { Tipos de inmunoterapia } \\
\text { /intervención farmacéu- } \\
\text { tica de pacientes con } \mathrm{CaP}\end{array}$ & [48] \\
\hline \multirow[t]{2}{*}{$\begin{array}{l}\text { Otras } \\
\text { fuentes }\end{array}$} & Vinculina & $\begin{array}{l}\text { Su expresión se asocia } \\
\text { con el aumento de la } \\
\text { proliferación de las } \\
\text { células tumorales en el } \\
\text { CaP }\end{array}$ & $\begin{array}{l}\text { Se requieren } \\
\text { mayores investi- } \\
\text { gaciones en otras } \\
\text { fuentes y tumores }\end{array}$ & $\begin{array}{l}\text { Posible Biomarcador } \\
\text { para el } \mathrm{CaP}\end{array}$ & [49] \\
\hline & $\begin{array}{l}\text { Caveolina-1 } \\
\text { y MRP1 }\end{array}$ & $\begin{array}{l}\text { Su aumento conduce a } \\
\text { la detención del creci- } \\
\text { miento y la inhibición de } \\
\text { la invasión celular del } \\
\mathrm{CaP}\end{array}$ & $\begin{array}{l}\text { Se requieren } \\
\text { mayores investi- } \\
\text { gaciones }\end{array}$ & $\begin{array}{l}\text { Diagnostico/Pronostico/ } \\
\text { Progresión de la enfer- } \\
\text { medad }\end{array}$ & $\begin{array}{l}52], \\
{[53]}\end{array}$ \\
\hline
\end{tabular}




\section{PROSTASOMAS Y CAP}

Los prostasomas fueron inicialmente caracterizados por Gunnar Ronquist a finales de 1970 [18]. La formación de los prostasomas se inicia desde una célula epitelial acinar de la próstata humana. Los prostasomas son enviados a la luz glandular por un evento exocitótico, que está precedido por la fusión con membranas adya- centes, también se puede ver que la vesícula de almacenamiento puede translocarse en su totalidad desde el interior de la célula hacia la luz acinar a través de la membrana plasmática, cuyo proceso se denomina como diacitosis. La vesícula de almacenamiento, corresponde a cuerpos multivesiculares de origen endosomal tardío [19] este proceso se representa en la Fig. 1.

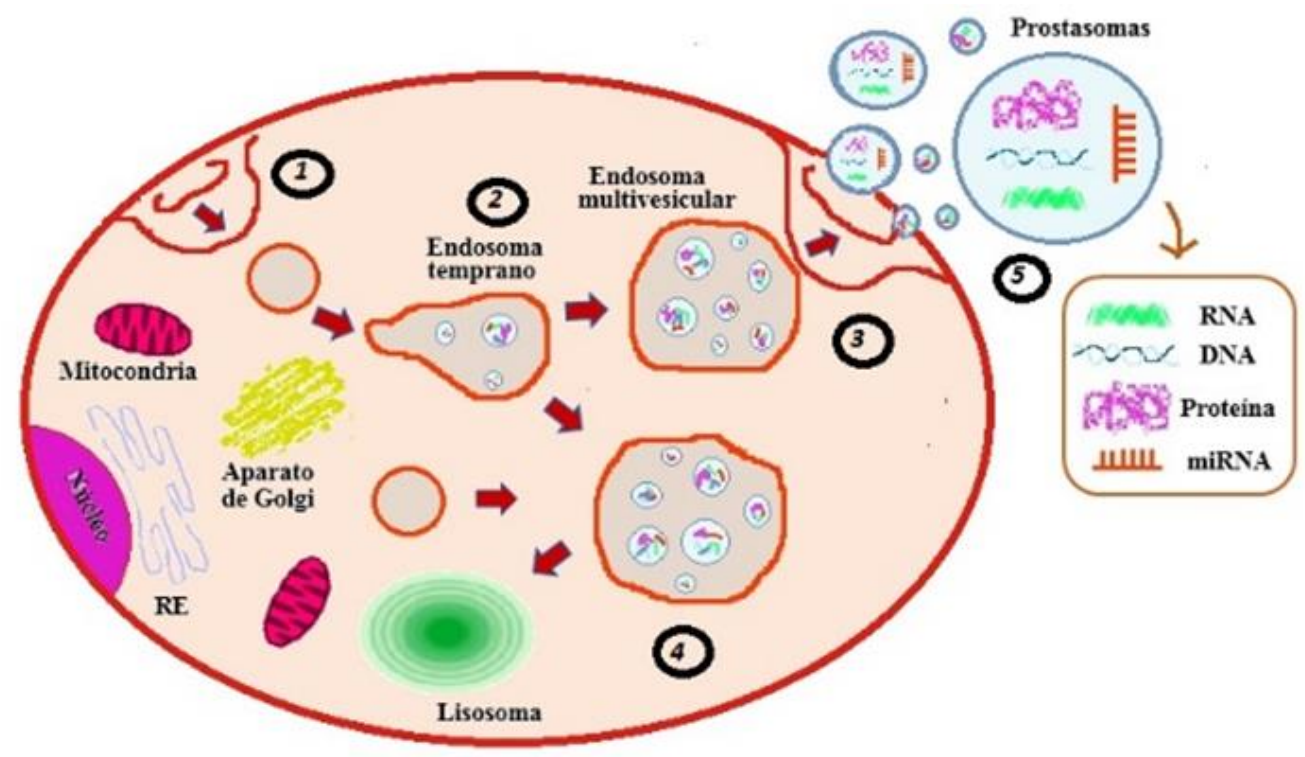

Fig.1. Formación de las VEs-prostasomas. Vía celular por la cual los prostasomas se se-cretan en el espacio extracelular. 1) Las vesículas se endocitan desde la superficie de la célula. 2) Estas vesículas transitan al endosoma temprano donde las invaginaciones del endosoma crean vesículas internas, dando como resultado un endosoma multivesicular (MVE). 3) El MVE puede dirigirse a la superficie de la célula 4) o dirigirse al lisosoma maduro en la cual se digerirá (destruirá) el conte-nido de la vesícula. 5) Por último, si con-tinua con la ruta de la exocitosis pasa a fusionarse con la membrana plasmática liberando su contenido, que incluye las vesículas internas que se convierten en prostasomas cuando se secretan desde la célula.

Fuente: Autores

Diferentes estudios mostraron que estos prostasomas poseen diversas funciones fisiológicas, generalmente relacionadas con las funciones de la célula parental, tienen la capacidad de llevar moléculas como lípidos o proteínas a los espermatozoides durante su viaje al ovocito, y poseen funciones tales como: actuar como mensajeros intercelulares entre las células secretoras de la próstata y las células espermáticas, ser una especie de "reservorio» que los espermatozoides pueden usar dependiendo de las condiciones del entorno [18], actuar en la regulación de la capacitación de las células espermáticas que se denomina como el proceso en el cual los espermatozoides alcanzan la capacidad de unirse a la zona pelúcida del ovocito, experimentan la reacción acrosómica y adquieren motilidad hiperactiva; esta motilidad hiperactivada es necesaria para que las células espermáticas se desprendan del epitelio oviductal, naden a través del moco allí presente y pasen la capa de células foliculares que rodean al ovocito, penetrando así la zona pelúcida, lo que conlleva a una correcta fertilización [15]. Aparte de los prostasomas, otros epitelios del tracto reproductor 
masculino también liberan VEs que se mezclan con prostasomas verdaderos $\mathrm{du}^{-}$ rante la emisión de semen [15], como el epitelio epididimal que produce VEs, que se cree que son eliminadas de la membrana plasmática de forma apocrina. Estas vesículas posteriormente se fusionan con la membrana plasmática espermática permitiendo la trasferencia de subconjuntos específicos de membrana y proteínas citosólicas a las células espermáticas.

El contenido proteico de los prostasomas es amplio; en un estudio realizado por Utleg et al, [20], donde se recolectaron muestras del eyaculado de 5 voluntarios sanos y se asilaron los prostasomas para la posterior caracterización de su proteoma, mediante shotgun Proteomics, identificaron un total de 139 proteínas, de las cuales 128 no habían sido descritas previamente como constituyentes prostasomales. Es importante mencionar que muchas de las proteínas reportadas en ese estudio son reguladas por andrógenos, tales como PSA, serina proteasa transmembrana tipo 2 (TMPRSS2), transglutaminasa específica de próstata, asimismo, algunas de ellas resultan muy específicas de la próstata de expresión abundante en este tejido, como PAP y antígeno prostático de células madre (PSCA) [15].

Las células tumorales y metastásicas de CaP pobremente diferenciadas tienen la capacidad de sintetizar y exportar mayor concentración de prostasomas, ya que la arquitectura de tejido se altera durante el proceso de carcinogénesis favoreciendo la liberación de prostasomas al espacio intersticial. Además, se conoce que las células tumorales tienden a explotar los sistemas fisiológicos del huésped con el fin de obtener apoyo en términos de, nutrición, crecimiento y metástasis. Parece que varias habilidades prostasomales, que se desarrollan para ayudar a las células espermáticas en la fertilización, también pueden ser promotoras en la transición de una célula normal a una neoplásica y ayudar a las células cancerígenas, mal diferen- ciadas, a sobrevivir y hacer metástasis [21].

\section{PRostasomas en fluido Sanguíneo}

El concepto general de que los exosomas aislados de la sangre puedan servir como posibles biomarcadores para la detección del cáncer fue recientemente ratificado para el cáncer de páncreas, ya que en este modelo se identificó que los exosomas de las células tumorales tienen contenidos específicos y únicos para el cáncer [22].

Como se mencionó anteriormente, las VEs secretadas por la membrana se desarrollan inicialmente dentro de los endosomas multivesiculares intracelulares, $\mathrm{du}^{-}$ rante este proceso, proteínas y ácidos nucleicos son encapsulados en los exosomas. Una vez se da la liberación en el espacio extracelular, dichas VEs entran en la circulación [17]. Particularmente, en CaP debido a la pérdida de la polaridad celular, lo cual favorece la liberación de los prostasomas al espacio intersticial y en consecuencia a la circulación [23], generando la posibilidad de aislarlos desde sangre para su posterior estudio.

Varios biomarcadores moleculares derivados de la sangre para la detección del CaP han estado disponibles a lo largo de los años [24]. Sin embargo, debido a su baja especificidad/sensibilidad y a lo pro- $^{-}$ metedor que pueden ser los prostasomas como posibles biomarcadores, se han realizado estudios con estas vesículas, los primeros intentos para demostrar la presencia de prostasomas en la sangre de un paciente con $\mathrm{CaP}$, se centraron en la detección de anticuerpos anti-prostasomas, ya que los prostasomas pueden estimular el sistema inmune induciendo la producción de autoanticuerpos, que pueden ser detectables en sangre de pacientes con $\mathrm{CaP}$, sin embargo, estos anticuerpos antiprostasomas son pocos específicos $\mathrm{y}$ además no permiten estratificar el CaP [25]. 
Sin embargo, se debe considerar que en el fluido sanguíneo no solo se encuentran prostasomas, si no también otras VEs que son secretadas activamente por la mayoría, sino todas, las células nucleadas, generando así una contaminación y por consecuencia convierte el proceso de aislamiento de los prostasomas en un procedimiento com- $^{-}$ plicado [17]. En un estudio realizado por Tavoosidana, et al, [23], donde propusieron una metodología para el aislamiento de los prostasomas, en la que la detección de estas microvesículas multiproteícas depende del reconocimiento simultáneo de cinco epítopes diferentes en al menos cuatro proteínas, aminopeptidasa N (CD13) y factor tisular (CD142), una glicoproteína asociada a la membrana celular que sirve como receptor y cofactor esencial para los factores VII y VIIa de la cascada de coagulación, mediante el uso de anticuerpos mono o policlonales diferentes, de manera que para detectar la suficiencia de los prostasomas en el plasma sanguíneo de pacientes con $\mathrm{CaP}$, realizan una modificación en el ensayo de ligadura de proximidad (PLA), el cual es un mecanismo para la detección sensible y especifica de proteínas en el que las moléculas de interés deben ser reconocidas por anticuerpos [26] y posteriormente cuantificadas mediante PCR en tiempo real.

El plasma sanguíneo fue recolectado de dos grupos de pacientes con CaP y sujetos controles, pareados por edad: el primer grupo incluyó muestras de 20 pacientes con un PSA de 94-2706 ng/ml y 20 controles con niveles de PSA inferiores a $2.5 \mathrm{ng} / \mathrm{ml}$. El segundo grupo estaba constituido por 59 pacientes con $\mathrm{CaP}$ y un PSA de 1.1 a 39.1 $\mathrm{ng} / \mathrm{ml}$ y se compararon con 20 controles con un PSA entre $1.7-14.8 \mathrm{ng} / \mathrm{ml}$ con resultados benignos de biopsia transrectal. Se detectaron niveles de prostasomas relevantes en las muestras de sangre de los pacientes con $\mathrm{CaP}$, hasta 7 veces más altos en comparación con las 20 muestras de los sujetos controles [23].
Al usar este ensayo, se demuestra con éxito que los prostasomas se pueden detectar en niveles elevados en el plasma sanguíneo de pacientes con $\mathrm{CaP}$, además los resultados también muestran que este análisis puede distinguir pacientes con puntajes de Gleason medio y alto de aquellos puntajes bajos, lo que permite correlacionar la agresividad del tumor. En conclusión, debido a su alta sensibilidad y especificidad para prostasomas en muestras de sangre, el ensayo realizado en este estudio es prometedor como un procedimiento para el aislamiento de estas vesículas, sin embargo, se deben continuar con los experimentos [23].

Por otro lado, la proteína antiapoptótica Survivin pertenece a una familia conocida de oncoproteínas, aislada de vesículas exosómicas derivadas de la sangre, expresada en la mayoría de tumores malignos [27], razón por la cual ha sido estudiada como posible biomarcador en distintos tipos de cáncer, entre ellos CaP. En un estudio realizado en muestras de sangre de pacientes afroamericanos con $\mathrm{CaP}$ reportaron un aumento significativo de esta proteína, en comparación de hombres pertenecientes a otros grupos raciales y a los sujetos controles [28]. Es necesario resaltar que el CaP suele ser más agresivo en afroamericanos que en varones de otros grupos étnicos; con este hallazgo se describen diferencias significativas en la biología tumoral de CaP entre grupos étnicos. Particularmente, los autores proponen que el aumento de la expresión de Survivina en VEs de pacientes afroamericanos con CaP puede influir en la agresividad del tumor y contribuir con la tasa de mortalidad observada en esta población [28]. Esta proteína podría servir como nuevo biomarcador y posible blanco terapéutico que podría tener utilidad clínica para mejorar la salud de pacientes con $\mathrm{CaP}$, ya que se ha demostrado que la Survivin se encuentra extracelularmente y está contenida en los exosomas [29], además que también se expresa en el $\mathrm{CaP}$ y su regulación sensibiliza las células 
del cáncer a los agentes quimioterapéuticos. Khan et al realizan un estudio utilizando [30] la técnica de ELISA y Western Blot respectivamente, para investigar la existencia de Survivina exosomal en el plasma de pacientes con $\mathrm{CaP}$ con una variedad de presentaciones de este y comparar la expresión de sus niveles en los exosomas encontrados en los sujetos controles y pacientes con Hiperplasia Prostática Benigna (HPB). Para ello, obtienen muestras de plasma sanguíneo de 10 voluntarios sanos y 28 muestras de pacientes con $\mathrm{CaP}$, se seleccionaron 10 muestras de bajo grado (Gleason 6) y 10 de alto grado (Gleason 9); además, recolectaron muestras de $8 \mathrm{pa}^{-}$ cientes con la enfermedad avanzada que participaban en quimioterapia y 20 mues- $^{-}$ tras de pacientes con HPB. En sus hallazgos, la Survivina fue detectable en todos los sujetos controles, pacientes con $\mathrm{CaP}$ y $\mathrm{HPB}$, sin embargo, sus resultados fueron diferentes para todos los casos, se encontró altamente expresada en los exosomas de pacientes con $\mathrm{CaP}$ que presentaban puntajes de Gleason de 6 y 9 , y en pacientes que habían recaído en la quimioterapia. No obstante, no hubo diferencias significativas en los niveles de Survivina entre los sujetos con puntuaciones de Gleason bajas o altas. Además, aunque los pacientes con HPB también contienen en sus exosomas Survivina, el nivel de expresión fue significativamente bajo en comparación con los hallados en pacientes con $\mathrm{CaP}$. En conclusión, este estudio demuestra que los niveles plasmáticos de Survivina exosómica puede ser una herramienta para el diagnóstico o monitorización del $\mathrm{CaP}$ [30].

Como el contenido del exosoma refleja su fuente celular, estos pueden contener proteínas oncogénicas o proteínas supresoras de tumores, por lo tanto, puede conducir a efectos positivos o negativos en relación con la progresión del cáncer. En esta investigación, realizada por Gabriel et al., en el 2013, se recolectan 30 muestras de pacientes con $\mathrm{CaP}$ en un estadio avanzado (T3 / T4) y antes de la prostatectomía, además de 8 hombres voluntarios sanos de 50 y 65 años de edad [31]. El CaP agresivo o metastásico se asocia con una reducción o pérdida de la expresión de PTEN, una potente proteína supresora de tumores, para lo cual los resultados es este estudio realizados por inmunotransferencia revelan una incorporación de PTEN en los exosomas que circulan en sangre de $\mathrm{pa}^{-}$ cientes con $\mathrm{CaP}$, pero, curiosamente, los sujetos sanos no muestran esta expresión en sus exosomas sanguíneos. Los resultados de este estudio mostraron un nuevo mecanismo por el cual las células cancerosas regulan la expresión de PTEN a través de exosomas; todo esto lleva a la conclusión de que la expresión de PTEN en los exosomas es una característica exclusiva de las células tumorales, además el estado de PTEN en pacientes con CaP podría ser un posible predictor del riesgo de metástasis o reaparición después de la prostatectomía. Sin embargo, se requieren mayores estudios para aprobar estos hallazgos [31].

Los microRNA extracelulares incrustados en exosomas circulantes pueden servir como biomarcadores pronósticos en el cáncer. En una investigación realizada por Huang et al en el año 2015, realizaron una secuenciación de RNA para identificar miRNA exosomal en 50 candidatos sanos y 36 pacientes con $\mathrm{CaP}$, de los cuales 23 pacientes eran resistentes a la castración (CRPC). Los miRNA se evaluaron mediante la técnica de PCR en tiempo real. El estudio identificó dos candidatos de miRNA (miR-1290 y miR-375), sin embargo, los miR -375 asociados con el cáncer han sido implicados en una variedad de carcinomas, incluidos el $\mathrm{CaP}$, la regulación de este se ha correlacionado con la metástasis del $\mathrm{CaP}$, pero también con la supervivencia de pacientes con cáncer esofágico; por otro lado, los miR-1290 tuvieron una mejor predicción en los resultados en comparación con miR-375, sin embargo, sus expresión aumentada en suero discrimina para el cáncer de páncreas en estadio bajo y también está involucrado en el cáncer de 
mama, en consecuencia su papel directo para el $\mathrm{CaP}$ permanece indeterminado. No obstante, en esta investigación observaron ciertas diferencias significativas en los resultados que sugieren los miRNA exosomales circundantes como posibles biomarcadores sensibles para el pronóstico de pacientes con CRPC [32].

En otro estudio realizado por Bryant et al [33] se planteó analizar los cambios en los microRNA (miR) circulantes como posibles biomarcadores para el diagnóstico, la estratificación y predicción del $\mathrm{CaP}$, para ello recolectaron 78 muestras de plasma sanguíneo de pacientes con CaP (12 sin metástasis, 51 M0 y 15 M1) y 28 de individuos control (PSA >10 ng/ml), y por medio de la técnica de PCR en tiempo real analizan 742 miRs utilizando las microvesículas circulantes (cMV). En sus resultados obtuvieron un total de $12 \mathrm{miR}$ que se cuantificaron diferencialmente, de los cuales 11 aumentaron significativamente en las cMV de los pacientes con $\mathrm{CaP}$ en comparación con las muestras control, mientras que la concentración de miR-181a -2 obtuvo una reducción. Posteriormente, analizan los cMV de 55 pacientes con $\mathrm{CaP}$ no metastásico para el cual identifican 10 miRs cuantificados diferencialmente, $9 \mathrm{miR}$ señalan un aumento significativo mientras que miR- 181a -2 expresa una disminución. Luego comparan los perfiles de miR en 16 pacientes con metástasis y 55 con $\mathrm{CaP}$ no metastásico, donde encuentran un total de $16 \mathrm{miR}$ cuantificados diferencialmente en pacientes con CaP metastásico en comparación con aquellos que poseen la enfermedad no metastásica; $15 \mathrm{miRs}$ señalan una concentración mayor, mientras que miR572 se expresó significativamente menor en varones con $\mathrm{CaP}$ metastásico frente al no metastásico. Esto lleva a la conclusión de que los cambios en las concentraciones de miR en pacientes con $\mathrm{CaP}$ pueden ser utilizados para el hallazgo del diagnóstico y para la estratificación del mismo [33].

Las células cancerígenas producen grandes cantidades de VEs, por lo que se ha propuesto que los exosomas tumorales influyen en la respuesta inmune y posiblemente contribuyen a la progresión del cáncer [34]. En una investigación realizada por Lundholm et al, [35] analizan en pacientes CRPC (pacientes con $\mathrm{CaP}$ resistentes a la castración) la capacidad de los exosomas derivados de células tumorales para regular negativamente la expresión de NKG2D el cual es un receptor de citotoxicidad que se expresa por una variedad de células inmunes, incluyendo las células NK, células NKT, CD8 y células T; su perdida en el cáncer es significativamente importante en la supresión inmune. Recolectan 18 muestras de sangre de pacientes con CRPC antes del inicio del tratamiento con quimioterapia y 8 muestras de sujetos sanos; usando la citometría de flujo, encuentran que los linfocitos circulantes de pacientes con CRPC expresan reducción de NKG2D además los exosomas derivados del tumor indujeron la regulación negativa de NKG2D en células T, NK y CD8 en comparación con los resultados de las muestras control. De acuerdo con estos hallazgos, la secreción de exosomas puede ser un mecanismo de escape inmune del tumor en la $\mathrm{CaP}$ [35].

\section{PROSTASOMAS EN ORINA}

La orina es un fluido de desecho corporal que se puede obtener fácilmente, por lo que es ideal para la determinación y el análisis de biomarcadores. Esta es una mezcla compleja de proteínas, sales, urea y metabolitos filtrados y secretados que pueden variar, no solo en situaciones fisiológicas, sino especialmente en enfermedades que afecten el sistema genitourinario [36].

En la actualidad, se sabe que hay presencia de prostasomas en fracciones de VEs aisladas de la orina mediante la detección de proteínas específicas de la próstata, entre las que se destacan el antígeno prostático específico de membrana (PSMA), la 
fosfatasa acida prostática y la trasglutaminasa prostática [17].

La orina contiene células tumorales prostáticas intactas y cuerpos apoptóticos derivados de dichas células. Las células y la mayoría de los cuerpos apoptóticos son considerablemente más grandes que los prostasomas, por lo tanto, pueden separarse fácilmente de estos mediante centrifugación diferencial [37], por esta razón la orina es una fuente importante para el estudio de biomarcadores asociados a prostasomas.

A pesar de que distintos tejidos dentro del sistema genitourinario masculino, como el renal, testicular, entre otros [38], contribuyen con las VEs encontradas en la orina, una ventaja particular de trabajar con este fluido como fuente de biomarcadores, es que frecuentemente se observa enriquecido en prostasomas. Probablemente, debido a las funciones especiales que tienen los prostasomas en la tumorogénesis del $\mathrm{CaP}$, la angiogénesis y la evasión de la respuesta inmune [36].

En un estudio [40] donde se analizó el proteoma de los exosomas urinarios, mediante la técnica de espectrometría de masas, con el objetivo de identificar proteínas expresadas diferencialmente en pa- $^{-}$ cientes con $\mathrm{CaP}$ en comparación con controles sanos; se aislaron los exosomas urinarios de 15 muestras control y 17 muestras de pacientes con $\mathrm{CaP}$, cuyo análisis reveló 246 proteínas diferencialmente $\mathrm{ex}^{-}$ presadas en los dos grupos, de las cuales 221 se encontraban sobreexpresadas en CaP. Particularmente, 37 de estas proteínas fueron seleccionadas con criterios específicos, tales como la sensibilidad, especificidad y veces de cambio en la expresión. Estas 37 proteínas juntas presentaban una especificidad del 100 \%. 17 de estas mostraron sensibilidades individuales superiores al $60 \%$. Aunque varias proteínas mos- $^{-}$ traron alta sensibilidad y especificidad para el $\mathrm{CaP}$ como biomarcadores individuales, combinándolas en una única prueba tiene el potencial para la diferenciación total del CaP contra los controles sanos. La mayor sensibilidad, $94 \%$ (16 de 17 pacien- $^{-}$ tes), se observó para la proteína transmembrana 256 (TM256). Asimismo, el complejo proteico LAMTOR también presentó muy alta especificidad en muestras de CaP. Estos resultados permiten demostrar el potencial uso de los exosomas urinarios en el diagnóstico y manejo clínico del CaP.

Otro estudio [16], donde se analizaron los exosomas urinarios de 9 pacientes con $\mathrm{CaP}$ dividiéndolos en los cuatro grupos siguientes: recién diagnosticados sin previo tratamiento, diagnosticados, bajo terapia de privación de andrógenos (ADT) y pacientes con metástasis óseas verificadas; a partir de una cantidad muy limitada de ARN exosómico y por medio de una PCR anidada lograron confirmar la presencia de dos conocidos biomarcadores de CaP; PCA3 y TMPRSS2:ERG. Las transcripciones de ARNm para el gen de fusión TMPRSS2: ERG se detectaron en los pacientes que tenían una puntuación de Gleason alta y altos niveles de PSA, y no en los pacientes con tumores de bajo riesgo, mientras que las transcripciones de PCA -3 se detectaron en todos los pacientes después de un masaje de próstata leve. Este estudio demostró que al incluir mRNAs y miRNAs exosómicos para la búsqueda de biomarcadores, no solo se contribuye con la detección de cáncer, sino también con la clasificación de la severidad del fenotipo tumoral y la respuesta al tratamiento.

Actualmente, se han identificado aproximadamente 1000 miRNAs en humanos y cada uno de ellos puede estar relacionado o apuntar a genes que influyen en vías de señalización importantes para la progresión de varios tipos de cáncer. Se ha comprobado que varios miRNA tienen su función alterada o se encuentran diferencialmente presentes en el CaP y parecen influir en la actividad antiapoptótica [33].

En un estudio poblacional [33] donde se recogieron muestras de orina de 135 hom- $^{-}$ bres posterior a un masaje prostático 
transrectal, se analizaron cinco miRNA seleccionados por medio de PCR-TR y se descubrió que miRNA-107 y miRNA-574$3 \mathrm{P}$ se sobreexpresaron significativamente en la orina de pacientes con $\mathrm{CaP}$ en comparación con los controles; ambos miRNAs podían identificar la presencia de $\mathrm{CaP}$ a partir de muestras de orina (índices de concordancia 0.66-0.74) y parecían más precisos que el PCA3 normalizado para el PSA urinario (índice de concordancia 0.61) lo cual nos deja como observación que un ensayo individual del nivel de concentración de miR-107, por ejemplo, puede ser una prueba de diagnóstico clínicamente útil para el CaP no metastásico.

\section{LÍQUIDO SEMINAL}

El Fluido Seminal (FS) está compuesto por altas concentraciones de proteínas, iones solubles y moléculas pequeñas, aproximadamente el $40 \%$ del FS es material prostático, liberado después de la eyaculación [40].

El FS tiene una serie de ventajas sobre la sangre y la orina en términos de su potencial como fuente de biomarcadores $\mathrm{es}^{-}$ pecíficos de CaP. En primer lugar, los constituyentes prostáticos están altamente enriquecidos en el FS en comparación con otros fluidos corporales. De hecho, el PSA se describió originalmente en FS, donde existe una concentración de aproximadamente 5-6 órdenes de magnitud mayor que en el suero sanguíneo. En segundo lugar, a diferencia de las células epiteliales prostáticas malignas y sus productos que solo ingresan a la circulación tras la trasgresión de las barreras del tejido sanguíneo, las células y sus secreciones se liberan de forma natural en el FS, tanto en las glándulas normales como en las malignas. Ambos factores sugieren que los biomarcadores se detectarían en FS antes que, en sangre, lo que destaca el potencial de este líquido para la detección temprana, incluidos los cambios premalignos. En tercer lugar, el
FS no solo contiene material libre de células de la próstata, sino también células tumorales detectables antes del diagnóstico de $\mathrm{CaP}$ basado en la biopsia, con la tasa de proliferación de estas células potencialmente valiosa en el control de pacientes con enfermedad de bajo grado en regímenes de vigilancia. [41]

Debido a que el FS es un medio molecular diverso y abundante, compuesto por ácidos nucleicos, proteínas, lípidos, azúcares, pequeños metabolitos e iones [42], permite que sea un candidato para la búsqueda de prostasomas como biomarcadores para el CaP. En los últimos años se han realizado intentos con diferentes técnicas para la identificación de biomarcadores específicos de $\mathrm{CaP}$. Recientemente en un estudio realizado por Carlsson et al, proceden al aislamiento de prostasomas para hacer una comparación entre las características funcionales $\mathrm{y}$ bioquímicas entre tres de estos de diferentes fuentes, entre ellas de líquido seminal. La muestra de semen se centrifugó durante 20 minutos para separar los espermatozoides y otras posibles células del plasma seminal, que luego se agruparon en 15 muestras, la citometría de flujo y ELISA fueron algunas de las técnicas utilizadas en esta investigación, para revelar que fuertes similitudes entre los prostasomas aislados de los tres tipos de muestras; una porción alta de algunas enzimas, colesterol y fosfolípidos fueron características comunes, además, las proteínas de membrana CD13, CD26, CD10 y CD46, previamente asociadas con el plasma seminal (SP), se encontrar en los tres tipos de prostasomas, asimismo, CD13 y DPPIV mostraron una concentración extremadamente mayor en este fluido. Estos resultados señalan que es posible encontrar prostasomas en diferentes fuentes, siendo SP bastante prometedor para futuras investigaciones en prostasomas [43].

Como se ha mencionado, los prostasomas cumplen una función en la regulación de los espermatozoides, la inmunosupre- 
sión y la progresión del CaP. Galectin-3 es una proteína de unión a carbohidratos multifuncional que se caracterizó inicialmente como un sustrato proteolítico para el PSA y se demostró que se asociaba con prostasomas en el semen humano [44], además participa en la inmunomodulación, interacciones celulares y en la progresión del cáncer, incluido el CaP [45]. En esta investigación realizada por Block et al, los ligandos galectin-3 candidatos en prostasomas se identificaron mediante espectrometría de masas, además, también se usaron otros métodos inmunoquímicos y bioquímicos para investigar la asociación de la proteína de unión Mac-2 (M2BP) con prostasomas. Cabe mencionar que la molécula de galectina-3 ejerce sus funciones mediante interacciones proteína-carbohidrato con ligandos glicoconjugados, estas interacciones se entrecruzan y generan ligandos que se unen a galectina-3 para inducir efectos posteriores [45]. Se obtuvo muestras de semen para realizar el aislamiento de los prostasomas, para lo cual los ligandos de unión a galectina 3 identificados en los resultados incluyen $\mathrm{M} 2 \mathrm{BP}$, CD26, PIP, peptidasa IV, olfactomedina-4 (OLF4) y seminogelinas I y II; M2BP se encontró en la superficie del prostasoma y en los espermatozoides. Los estudios indicaron que M2BP es de alta importancia funcional con los prostasomas, lo que siembra una base para investigaciones futuras sobre la progresión del CaP respectivamente [45].

En otro estudio publicado por MJ et al, donde estudiaron la precisión pronóstica o diagnóstica para el $\mathrm{CaP}$ por medio del FS, se analizan una cohorte de muestras de 152 hombres sospechosos de tener CaP por los resultados iniciales de un PSA aumentado y/o un tacto rectal anormal; los pacientes se clasificaron en dos grupos: alto riesgo y bajo riesgo [46]. Se evaluó la precisión diagnóstica de PCA3, un RNA no codificante asociado a $\mathrm{CaP}$ y Hepsin, una proteasa transmembrana previamente asociada con CaP. Una combinación de Hepsin, PCA3 y PSA sérico mejora la predic- ción del estado del $\mathrm{CaP}$ y el riesgo clínico con mayor precisión que el PSA sérico solo. Además, la integración de los marcadores de miRNA con PCA3 y Hepsin mejoró aún más la especificidad diagnóstica y la predicción del riesgo [46].

En una nueva investigación realizada por Dubois et al, primeramente, se recolecta plasma seminal de 48 varones con $\mathrm{CaP}$ para evaluar la expresión de proteínas de chromogranin (cg) en prostasomas y VEs de células malignas de la próstata. Por medio de la técnica de ELISA se observaron patrones divergentes de péptidos de chromogranin al comparar prostasomas $\mathrm{y}$ VEs, lo que indican un cambio fenotípico [47]. El estudio demostró un mosaico de péptidos pertenecientes a miembros clásicos de la familia de proteínas de graninas en la superficie no solo de prostasomas sino también de VEs, además la expresión de los prostasomas difería significativamente del hallado en las VEs. Por lo tanto, se llega a la conclusión de que los péptidos de cgA serían detectables preferiblemente en prostasomas provenientes de células no malignas, mientras que los péptidos cgB se detectaron más fácilmente en las VEs de células malignas; estas Cromogranina no solo de prostasomas sino también de VEs derivadas del $\mathrm{CaP}$ pueden ser de gran importancia para el diseño de nuevos ensayos sobre el grado de malignidad del $\mathrm{CaP}$ [47].

Las propiedades del CaP cambian drásticamente cuando las células se vuelven independientes de andrógenos y se produce la metástasis. Se ha revelado que estas células metastásicas del $\mathrm{CaP}$ pueden producir prostasomas y exportarlas al espacio extracelular; por lo tanto, se cree que los prostasomas producidos por las propias células de $\mathrm{CaP}$ son los principales actores en el proceso maligno y que la resistencia contra el sistema inmune es una característica central en el proceso de selección [48]. En el estudio realizado por Babiker et al, investigaron la expresión y la función de las proteínas quinasas prostasómicas y 
la ATPasa, para lo cual cultivan líneas celulares de $\mathrm{CaP}$ y también aíslan prostasomas de 30 muestras de líquido seminal de hombres remitidos a la clínica de fertilidad en el Hospital Universitario de Uppsala; las funciones de la proteína quinasa en prostasomas aislados se analizaron mediante la fosforilación de sustratos, también se evalúa la actividad de la ATPasa, además utilizan la citometría de flujo para verificar estas expresiones. Los resultados de esta investigación demuestran que todos los prostasomas de origen celular poseen actividad significativamente superior de proteína quinasa en comparación con los seminales, lo que resulta en una mayor fosforilación, tanto de sustratos exógenos como endógenos. También se encontró que las prostasomas de origen metastásico tenían una actividad de ATPasa más baja, además, el componente $\mathrm{C} 3$ y el fibrinógeno (dos proteínas cuyas actividades están moduladas por la fosforilación) obtuvieron una expresión relevante en la fosforilación. Si la sobreexpresión de proteínas quinasas en las células de $\mathrm{CaP}$ metastásicas se pudiera controlar o contrarrestar, podría potenciar otros tipos de inmunoterapia, todos estos resultados llevan a la búsqueda de nuevos objetivos para la intervención farmacéutica de pacientes con $\mathrm{CaP}$ [48].

\section{OTRAS FUENTES ASOCIADAS A PROSTASOMAS}

Existen otras fuentes como posibles biomarcadores asociadas a prostasomas, se conoce que las vesículas extracelulares además de estar circulando en diferentes fluidos corporales, poseen diferentes moléculas, entre ellas RNA [38]. Estas VE realizan acciones biológicas en lugares distantes de su origen, interactúan con otras células por diferentes mecanismos como la unión de receptores en la membrana celular desencadenando vías de señalización intracelulares y liberando así su contenido [49].
Debido a que las VE son valiosas herramientas para la detección, el pronóstico y tratamiento del cáncer, se realizó el análisis de microvesículas liberadas al entorno extracelular utilizando líneas celulares del $\mathrm{CaP}$ metastásico $\mathrm{PC}-3$, por medio de dos técnicas, la cromatografía líquida nanocapilar y espectrometría de masas; lograron identificar 266 proteínas con secuencias peptídicas, debido a que varias de estas proteínas se han identificado anteriormente en exosomas, esto indica que las vesículas PC-3 tienen características en común [49].

En particular, se mostraron tres proteínas de interés, CDCP1, la tetraspanin CD151 y CD147, además de otras proteínas como la TCTP y la neuropilina, todas estas asociadas y / o desreguladas en el $\mathrm{CaP}$; por otro lado, fue muy relevante encontrar niveles abundantes de vinculina (proteína de la familia actina involucrada en la interacción entre el citoesqueleto y la matriz extracelular) en las microvesículas de PC-3. Esta última parece ser un biomarcador prometedor ya que su expresión se asocia con el aumento de la proliferación de las células tumorales en el CaP [49].

La Vigilancia Activa (VA) del $\mathrm{CaP}$ en estadio temprano se ha venido implementando en la última década, ya que el diagnóstico temprano y el tratamiento curativo contribuyen con la supervivencia de hombres con cánceres de riesgo desfavorable, sin embargo, existen preocupaciones significativas con respecto al sobrediagnóstico y el sobretratamiento de hombres con $\mathrm{CaP}$ de riesgo menor. Aunque las cohortes de VA publicadas difieren según el protocolo utilizado, las tasas de metástasis de la enfermedad y la mortalidad específica por $\mathrm{CaP}$ son extremadamente bajas en el mediano plazo (5-10años). Tales resultados parecen estar estrechamente relacionados con los criterios específicos del programa para la selección, monitoreo e intervención, sugiriendo que la VA al igual que otras estrategias de manejo podrían ser más eficientes para la correcta estratificación 
de pacientes con $\mathrm{CaP}$. Con el fin de refinar la estratificación del riesgo, el monitoreo individual a través de biomarcadores específicos podrían ser una opción, si estos marcadores son de tipo circulantes, se evade la desventaja asociada al submuestreo inherente a la biopsia prostática [50], [51].

Ya que algunos estudios han sugerido los prostasomas como posibles indicadores de la progresión del cáncer, se encontró que Caveolina $\left(\mathrm{Cav}^{-1}\right)$ es una proteína de membrana que se unen fuertemente al colesterol, su expresión aumentada está acompañada por la adquisición de fenotipo de resistencia a múltiples fármacos (MDR) en las células cancerígenas de la próstata [52]. Debido a que el CaP también es resistente a una amplia gama de agentes antineoplásicos, se ha observado en un estudio realizado por Pellinen et al, en 435 mues- $^{-}$ tras de tejido prostático tomadas a pacientes con grados primarios y secundarios de la escala de Gleason, que $\mathrm{Cav}^{-1}$ en prostasomas de células tumorales de $\mathrm{CaP}$ junto con CD59, como un marcador específico de estas vesículas, reflejan prometedores resultados, $\mathrm{Cav}^{-1}$ condujo a la detención del crecimiento y la inhibición de la invasión celular en líneas celulares de $\mathrm{CaP}$ lo cual podrían servir como diagnostico o pronostico del $\mathrm{CaP}$ además de evaluar su progresión en pacientes que ya padezcan la enfermedad [52].

En otro estudio realizado en tres líneas celulares de próstata (normal, andrógenosensible y andrógeno-independiente) derivadas de metástasis a ganglios linfáticos y hueso, respectivamente, con el fin de comprender su posible papel en la quimiorresistencia al $\mathrm{CaP}$, se muestra que la proteína de resistencia Multirresistente 1 (MRP1) se encuentra en fracciones de balsa lipídica de células tumorales y que el número de caveolas aumenta con la adquisición de malignidad. MRP1 no solo se encontró en la membrana plasmática asociada con las balsas lipídicas sino también en las acumulaciones citoplásmicas que se localizan con los marcadores de prostasoma Caveolin-1 y CD59 [53]. Debido a que las tres líneas celulares mostraron prostasomas y que existe presencia de MRP1 en estos, los prostasomas podrían servir como un predictor de malignidad en $\mathrm{CaP}$. Además, se llegó a la conclusión que tal vez haya dos poblaciones diferentes de células, aquellas menos agresivas que no expresa MRP1 en prostasomas y aquellas que tienen MRP1 en prostasomas localizado siendo estas más agresivas. Se puede concluir que tanto CD59 como Cav-1 podrían actuar como marcadores de prostasoma cuando las células adquieren malignidad y que las células cancerosas que tienen más prostasomas tendrían menos MRP1 en la membrana plasmática [53].

Debido a la transferencia de material envuelto en microvesículas desde la células tumorales a las células normales, se realizó un estudio con el objetivo de identificar la expresión de los genes específicos de la próstata en células normales de médula cultivadas con células de CaP [54]. Se recolectaron 11 tejidos de pacientes con $\mathrm{CaP}, 1$ normal y muestras de medula ósea de voluntarios sanos; los resultados arrojan una expresión génica significativamente $\mathrm{au}^{-}$ mentada, se encontró que las microvesículas del CaP podrían entrar en monocitos circulantes, células madre u otras células, logrando así alterar el fenotipo de una célula del $\mathrm{CaP}$. Todo esto representa una oportunidad para nuevas estrategias terapéuticas, tales como anticuerpos, para bloquear la liberación de microvesículas de células cancerosas [54].

\section{CONCLUSIONES}

La poca especificidad y sensibilidad del PSA como marcador diagnóstico para el $\mathrm{CaP}$ ha llevado a la comunidad científica a una intensa búsqueda de nuevos biomarcadores en fuentes biológicas como la orina, la sangre, fluidos prostáticos y otros. La composición molecular de las vesículas 
extracelulares propias de la próstata ha revelado proteínas como posibles candidatas a biomarcadores para el diagnóstico, estratificación y pronóstico del CaP. El desarrollo de estos marcadores requiere el análisis en grandes cohortes de pacientes, además, de aumentar la pureza de los aislamientos de los prostasomas contribuyentes en sangre, orina o fluidos prostáticos.

Una ventaja de los prostasomas muestreados a partir de sangre $u$ orina es que representan el estado general del organismo y el desarrollo del CaP, además de ser mínimamente invasivos.

La composición molecular de los prostasomas como proteínas y moléculas de ARN, entre otras, y la capacidad de estos de ser estables y que el ARN dentro de su lumen pueda resistir la ARNasa exógena, proporciona la capacidad para influir en el desarrollo y la metástasis del CaP. Por lo tanto, los prostasomas pueden considerarse abundantes en posibles marcadores para el diagnóstico/pronóstico del cáncer de próstata y servir como lectura para el estado de sus células de origen.

Sin embargo, las moléculas asociadas con prostasomas ya identificadas deben probarse en estudios de cohortes mayores de pacientes, para determinar su especificidad y sensibilidad en el diagnóstico de $\mathrm{CaP}$; de igual manera, se debe ampliar el campo de investigación en metodologías más eficientes para el aislamiento de los prostasomas en los distintos fluidos biológicos, para su posterior estudio en estos, ya que esta es una de las principales debilidades que los prostasomas tienen.

\section{AGRADECIMIENTOS}

Agradecemos la financiación otorgada por el Instituto Tecnológico Metropolitano, a través del proyecto de investigación P10240.

\section{REFERENCIAS}

[1] B. Pullar and N. Shah, "Prostate cancer," Surg., vol. 34, no. 10, pp. 505-511, Oct. 2016.

[2] J. Ferlay et al., "GLOBOCAN 2012: Estimated Cancer Incidence, Mortality and Prevalence Worldwide in 2012 v1.0," International Agency for Research on Cancer, 2012. [Online]. Available: http://publications.iarc.fr/Databases/IarcCancerbases/GLOBOCAN-2012-EstimatedCancer-Incidence-Mortality-And-PrevalenceWorldwide-In-2012-V1.0-2012.

[3] Organización Mundial de la Salud and Organización Panamericana de la Salud, "Plataforma de información en salud de las Américas," OPS, 2017. [Online]. Available: https://www.paho.org/hq/index.php?option $=\mathrm{c}$ om_tabs\&view $=$ article\&id $=2151 \&$ Itemid $=36$ $32 \&$ lang $=$ es.

[4] A. R. Lima, M. de L. Bastos, M. Carvalho, and P. Guedes de Pinho, "Biomarker Discovery in Human Prostate Cancer: an Update in Metabolomics Studies," Transl. Oncol., vol. 9, no. 4, pp. 357-370, Aug. 2016.

[5] Y. A. Goo and D. R. Goodlett, "Advances in proteomic prostate cancer biomarker discovery," J. Proteomics, vol. 73, no. 10, pp. 1839-1850, Sep. 2010.

[6] R. A. Castillejos-Molina and F. B. GabilondoNavarro, "Prostate Cancer," Salud Publica Mex., vol. 58, no. 2, pp. 279-284, 2016.

[7] Instituto Nacional del Cáncer, "Análisis del antígeno prostático específico (PSA) National Cancer Institute." [Online]. Available:

https://www.cancer.gov/espanol/tipos/prostat a/hoja-informativa-psa.

[8] A. Heidenreich et al., "EAU Guidelines on Prostate Cancer. Part 1: Screening, Diagnosis, and Treatment of Clinically Localised Disease," Eur. Urol., vol. 59, no. 1, pp. 61-71, Jan. 2011.

[9] S. Loeb and W. J. Catalona, "The Prostate Health Index: a new test for the detection of prostate cancer," Ther. Adv. Urol., vol. 6, no. 2, pp. 74-77, Apr. 2014.

[10] M. Lazzeri et al., "Relationship of Chronic Histologic Prostatic Inflammation in Biopsy Specimens With Serum Isoform [-2]proPSA (p2PSA), \%p2PSA, and Prostate Health Index in Men With a Total Prostate-specific Antigen of $4-10 \mathrm{ng} / \mathrm{mL}$ and Normal Digital Rectal Examination," Urology, vol. 83, no. 3, pp. 606-612, Mar. 2014.

[11] D. Hessels et al., "DD3PCA3-based Molecular Urine Analysis for the Diagnosis of Prostate Cancer," Eur. Urol., vol. 44, no. 1, pp. 8-16, Jul. 2003. 
[12] Y. Fradet et al., "uPM3, a new molecular urine test for the detection of prostate cancer," Urology, vol. 64, no. 2, pp. 311-315, Aug. 2004.

[13] R. Wang, A. M. Chinnaiyan, R. L. Dunn, K. J. Wojno, and J. T. Wei, "Rational approach to implementation of prostate cancer antigen 3 into clinical care," Cancer, vol. 115, no. 17, pp. 3879-3886, Sep. 2009.

[14] M. A. Álvarez-Blanco, P. M. Escudero-de losRíos, and H.-T. N, "Cáncer de próstata," Rev. Mex. Urol., vol. 68, no. 4, pp. 245-254, 2008.

[15] M. Aalberts, T. A. E. Stout, and W. Stoorvogel, "Prostasomes: extracellular vesicles from the prostate," Reproduction, vol. 147, no. 1, pp. R1-R14, Jan. 2014.

[16] J. Nilsson et al., "Prostate cancer-derived urine exosomes: a novel approach to biomarkers for prostate cancer," $\mathrm{Br}$. J. Cancer, vol. 100, no. 10, pp. 1603-1607, May 2009.

[17] C. Zijlstra and W. Stoorvogel, "Prostasomes as a source of diagnostic biomarkers for prostate cancer," J. Clin. Invest., vol. 126, no. 4, pp. 1144-1151, Apr. 2016.

[18] F. Saez and R. Sullivan, "Prostasomes, posttesticular sperm maturation and fertility," Front. Biosci. (Landmark Ed., vol. 21, pp. 1464-73, 2016.

[19] G. Ronquist, "Prostasomes are mediators of intercellular communication: from basic research to clinical implications," J. Intern. Med., vol. 271, no. 4, pp. 400-413, Apr. 2012.

[20] A. G. Utleg et al., "Proteomic analysis of human prostasomes," Prostate, vol. 56, no. 2, pp. 150-161, Jul. 2003.

[21] K. G. Ronquist, G. Ronquist, A. Larsson, and L. Carlsson, "Proteomic analysis of prostate cancer metastasis-derived prostasomes," Anticancer Res., vol. 30, no. 2, pp. 285-290, Feb. 2010.

[22] S. A. Melo et al., "Glypican-1 identifies cancer exosomes and detects early pancreatic cancer," Nature, vol. 523, no. 7559, pp. 177182, Jul. 2015.

[23] G. Tavoosidana et al., "Multiple recognition assay reveals prostasomes as promising plasma biomarkers for prostate cancer," Proc. Natl. Acad. Sci., vol. 108, no. 21, pp. 8809-8814, May 2011.

[24] D. a Sartori and D. W. Chan, "Biomarkers in prostate cancer," Curr. Opin. Oncol., vol. 26, no. 3, pp. 259-264, May 2014.

[25] B. O. Nilsson, L. Carlsson, A. Larsson, and G. Ronquist, "Autoantibodies to Prostasomes as New Markers for Prostate Cancer," Ups. J. Med. Sci., vol. 106, no. 1, pp. 43-50, Jan. 2001.

[26] S. Fredriksson et al., "Protein detection using proximity-dependent DNA ligation assays,"
Nat. Biotechnol., vol. 20, no. 5, pp. 473-477, May 2002.

[27] J. Gu, L. Ren, X. Wang, C. Qu, and Y. Zhang, "Expression of livin, survivin and caspase-3 in prostatic cancer and their clinical significance.," Int. J. Clin. Exp. Pathol., vol. 8, no. 11, pp. 14034-9, 2015.

[28] S. Khan et al., "Racial differences in the expression of inhibitors of apoptosis (IAP) proteins in extracellular vesicles (EV) from prostate cancer patients," PLoS One, vol. 12, no. 10, p. e0183122, Oct. 2017.

[29] S. Khan, J. M. S. Jutzy, J. R. Aspe, D. W. McGregor, J. W. Neidigh, and N. R. Wall, "Survivin is released from cancer cells via exosomes," Apoptosis, vol. 16, no. 1, pp. 1-12, Jan. 2011.

[30] S. Khan et al., "Plasma-Derived Exosomal Survivin, a Plausible Biomarker for Early Detection of Prostate Cancer," PLoS One, vol. 7, no. 10, p. e46737, Oct. 2012.

[31] K. Gabriel et al., "Regulation of the Tumor Suppressor PTEN through Exosomes: A Diagnostic Potential for Prostate Cancer," PLoS One, vol. 8, no. 7, p. e70047, Jul. 2013.

[32] X. Huang et al., "Exosomal miR-1290 and miR-375 as Prognostic Markers in Castration-resistant Prostate Cancer," Eur. Urol., vol. 67, no. 1, pp. 33-41, Jan. 2015.

[33] R. J. Bryant et al., "Changes in circulating microRNA levels associated with prostate cancer," Br. J. Cancer, vol. 106, no. 4, pp. 768-774, Feb. 2012.

[34] P. Filipazzi, M. Bürdek, A. Villa, L. Rivoltini, and V. Huber, "Recent advances on the role of tumor exosomes in immunosuppression and disease progression," Semin. Cancer Biol., vol. 22, no. 4, pp. 342-349, Aug. 2012.

[35] M. Lundholm et al., "Prostate TumorDerived Exosomes Down-Regulate NKG2D Expression on Natural Killer Cells and CD8+ T Cells: Mechanism of Immune Evasion," PLoS One, vol. 9, no. 9, p. e108925, Sep. 2014.

[36] A. Gámez-Valero, S. Lozano-Ramos, I. Bancu, R. Lauzurica-Valdemoros, and F. E. BorrÃ s, "Urinary Extracellular Vesicles as Source of Biomarkers in Kidney Diseases," Front. Immunol., vol. 6, p. 6, Jan. 2015.

[37] S. Principe et al., "In-depth proteomic analyses of exosomes isolated from expressed prostatic secretions in urine," Proteomics, vol. 13, no. 10-11, pp. 1667-1671, May 2013.

[38] J. K. O. Skog, X. O. Breakefield, D. Brown, K. C. Miranda, and L. M. Russo, "Uso de microvesículas en el diagnóstico, pronóstico y tratamiento de enfermedades y afecciones médicas," 2446 301, 2013.

[39] A. Øverbye et al., "Identification of prostate cancer biomarkers in urinary exosomes," 
Prostasomas: búsqueda de biomarcadores para la detección temprana del cáncer prostático

Oncotarget, vol. 6, no. 30, pp. 30357-30376, Oct. 2015.

[40] R. W. Cotton and M. B. Fisher, "Review: Properties of sperm and seminal fluid, informed by research on reproduction and contraception," Forensic Sci. Int. Genet., vol. 18, pp. 66-77, Sep. 2015.

[41] M. J. Roberts, R. S. Richards, R. A. Gardiner, and L. A. Selth, "Seminal fluid: a useful source of prostate cancer biomarkers?," Biomark. Med., vol. 9, no. 2, pp. 77-80, Feb. 2015.

[42] A. Lo Cicero, P. D. Stahl, and G. Raposo, "Extracellular vesicles shuffling intercellular messages: for good or for bad," Curr. Opin. Cell Biol., vol. 35, pp. 69-77, Aug. 2015.

[43] L. Carlsson, O. Nilsson, A. Larsson, M. Stridsberg, G. Sahlén, and G. Ronquist, "Characteristics of human prostasomes isolated from three different sources," Prostate, vol. 54, no. 4, pp. 322-330, Mar. 2003.

[44] M. R. Kovak, S. Saraswati, S. D. Goddard, and A. B. Diekman, "Proteomic identification of galectin-3 binding ligands and characterization of galectin-3 proteolytic cleavage in human prostasomes," Andrology, vol. 1, no. 5, pp. 682-691, Sep. 2013.

[45] A. S. Block, S. Saraswati, C. F. Lichti, M. Mahadevan, and A. B. Diekman, "Copurification of Mac-2 binding protein with galectin-3 and association with prostasomes in human semen," Prostate, vol. 71, no. 7, pp. 711-721, May 2011.

[46] M. J. Roberts et al., "Diagnostic performance of expression of PCA3, Hepsin and miR biomarkers inejaculate in combination with serum PSA for the detection of prostate cancer," Prostate, vol. 75, no. 5, pp. 539-549, Apr. 2015.

[47] L. Dubois et al., "Malignant cell-derived extracellular vesicles express different chromogranin epitopes compared to prostasomes," Prostate, vol. 75, no. 10, pp. 1063-1073, Jul. 2015.

[48] A. A. Babiker, G. Ronquist, B. Nilsson, and K. N. Ekdahl, "Overexpression of ectoprotein kinases in prostasomes of metastatic cell origin," Prostate, vol. 66, no. 7, pp. 675686, May 2006.

[49] J. H. Teixeira, A. M. Silva, M. I. Almeida, M. A. Barbosa, and S. G. Santos, "Circulating extracellular vesicles: Their role in tissue repair and regeneration," Transfus. Apher. Sci., vol. 55, no. 1, pp. 53-61, 2016.

[50] J. J. Tosoian, H. B. Carter, A. Lepor, and S. Loeb, "Active surveillance for prostate cancer: current evidence and contemporary state of practice," Nat. Rev. Urol., vol. 13, no. 4, pp. 205-215, Apr. 2016.

[51] S. P. Basourakos et al., "Baseline and longitudinal plasma caveolin-1 level as a biomarker in active surveillance for earlystage prostate cancer," BJU Int., vol. 121, no. 1, pp. 69-76, Jan. 2018.

[52] T. Pellinen et al., "ITGB1-dependent upregulation of Caveolin-1 switches TGFB signalling from tumour-suppressive to oncogenic in prostate cancer," Sci. Rep., vol. 8, no. 1, p. 2338, Dec. 2018.

[53] P. Gimenez-Bonafe et al., "Multidrug resistance protein 1 localization in lipid raft domains and prostasomes in prostate cancer cell lines," Onco. Targets. Ther., vol. 7, pp. 2215-2225, Dec. 2014.

[54] J. F. Renzulli et al., "Microvesicle Induction of Prostate Specific Gene Expression in Normal Human Bone Marrow Cells," J. Urol., vol. 184, no. 5, pp. 2165-2171, Nov. 2010 .. 